\title{
Texture Characterization Including Warm/Cool Sensation Using Force-, Light-, and Temperature-Sensitive Micro-electromechanical Systems Sensor
}

\author{
Fumiya Sato, Kenta Takahashi, Takashi Abe, \\ Masanori Okuyama, ${ }^{1}$ Haruo Noma, ${ }^{2}$ and Masayuki Sohgawa* \\ Niigata University, 8050 Ikarashi 2-no-cho, Nishi-ku, Niigata 950-2181, Japan \\ ${ }^{1}$ Osaka University, 1-3 Machikaneyama-cho, Toyonaka, Osaka 560-8531, Japan \\ ${ }^{2}$ Ritsumeikan University, 1-1-1 Nojihigashi, Kusatsu, Shiga 525-8577, Japan
}

(Received August 1, 2016; accepted February 23, 2017)

Keywords: texture measurement, warm/cool sensation, tactile sensor, multimodal measurement

In this paper, we report on texture measurements for an object, including tactile, warm/cool, and light sensations using a multimodal micro-electromechanical systems (MEMS) sensor. This MEMS sensor can detect force as a resistance change of the strain gauge on a microcantilever and light as an impedance change of a Si layer by a photoconductive effect. The sensor, which was maintained near human body temperature using a heater, can also detect a temperature drop after heat transfer from the sensor to a contacted object as a resistance change of the strain gauge or an impedance change of the Si layer. In this work, three different materials (copper, acrylic, and wood) were chosen as the target objects for the measurement of surface texture, and they were characterized on the basis of the differences in sensor outputs in active sensing experiments (approaching and pressing of the sensor with a probe light) on the sensor surface. In the proximity process, the impedance change of the Si layer depends on the surface reflectivity of the objects. After the object is touched by the sensor surface, the impedance of the Si layer and the resistance of the strain gauge of the sensor increase with the change in temperature caused by heat transfer. Furthermore, the resistance of the strain gauge decreases with the deformation of the cantilever caused by the pressing force from each object. Therefore, it is demonstrated that surface texture including the mechanical, optical, and thermal characteristics of various materials can be evaluated by active sensing using the proposed MEMS multimodal sensor.

\section{Introduction}

In recent years, texture designs have become increasing important for high-value-added products, such as clothes, furniture, and automobiles.(1) Generally, the textures of these products are characterized by human sensory evaluation. ${ }^{(2)}$ However, there is a lack of quantitative and reproducible data in sensory evaluation, because the evaluation result depends on human senses and the physical conditions of the subjects. For the non-sensation-based measurement of texture, some methods using a sensor device have been proposed. Mazid and Russell have measured texture as surface roughness using a tactile sensor, ${ }^{(3)}$ and Kuramitsu et al. have reported a texture 
measurement method based on the difference in surface friction using a human finger model.(4) However, the human tactile sense is intricately affected by roughness, coolness, moistness, hardness, and visual senses.(5) Hence, a multimodal sensing of various physical and optical properties is needed to characterize an object's texture more accurately. In our previous work, we developed a multi-axial micro-electromechanical systems (MEMS) tactile sensor that can detect normal and shear forces using a strain gauge film on microcantilevers ${ }^{(6)}$ and demonstrated that the surface texture of various objects can be evaluated by the active touching of this sensor. The feature quantities for an object depending on surface texture, including hardness/softness, friction, surface roughness, and other physical characteristics, have been extracted from the sensor output change in the active touching measurement and analyzed by principal component analysis for the classification of an object using the features of the surface texture. ${ }^{(7)}$ Moreover, this tactile sensor has a light sensing function as an impedance change of the Si substrate because of the photoconductive effect. ${ }^{(8)}$ We have already proposed a measurement method for the visual and tactile textures of an object using this sensor. ${ }^{(9)}$

As mentioned in Ref. 10, the warm/cool sensation is also one of the most important properties in human texture perception. Humans perceive the warm/cool sensation not only by the temperature difference of the object but also the rate of change in temperature during touching by heat transfer from the skin to the object. ${ }^{(11)}$ Hence, the warm/cool sensation should be measured to characterize the surface texture more accurately. Normally, the electrical conduction characteristics of materials depend on temperature; thus, it is suggested that the resistance of the strain gauge and the impedance of the Si layer in our sensor are sensitive to strain and light as well as to temperature. In this work, we explore the possibility of measuring texture including tactile, visual, and warm/cool sensations using the developed MEMS sensor.

\section{Structure and Detection Principle}

\subsection{Structure of the multimodal MEMS sensor}

Figures 1(a) and 1(b) show the concept of the multimodal MEMS sensor and a cross-sectional structure of the microcantilever, respectively. The microcantilevers of the sensor ( $290 \mu \mathrm{m}$ long, $200 \mu \mathrm{m}$ wide, $3 \mu \mathrm{m}$ thick) were fabricated by a surface MEMS process on a Si-on-insulator (SOI) wafer ${ }^{(12)}$ and have an inclined shape because of the residual stress in the Cr layer. They

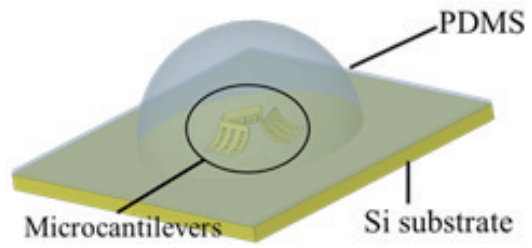

(a)

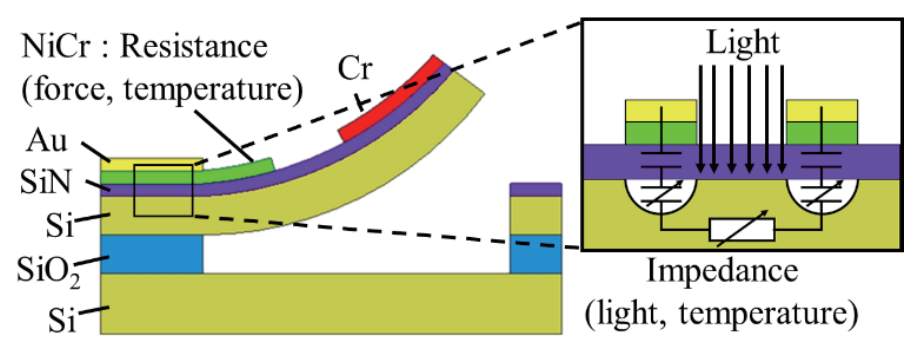

(b)

Fig. 1. (Color online) Conceptual illustrations of (a) the multimodal MEMS sensor and (b) a cross-sectional structure of the microcantilever. 
were embedded in a polydimethylsiloxane (PDMS) layer (about $40 \mu \mathrm{m}$ ) by spin-coating, and then a hemispherical-shaped PDMS (diameter: $2.1 \mathrm{~mm}$ ) cast by a plastic mold form was put over the microcantilevers as the contact part of the sensor. There are thin-film strain gauges on each microcantilever to detect the deflection as a resistance change. Furthermore, the AC impedance between the electrodes includes resistance and depletion layer capacitance in Si depending on light intensity, as shown in the enlarged illustration in Fig. 1(b). Three microcantilevers are located within $1 \mathrm{~mm} \phi$ at $120^{\circ}$ intervals. $\operatorname{SiN}(500 \mathrm{~nm}), \operatorname{NiCr}(50 \mathrm{~nm}), \mathrm{Cr}(200 \mathrm{~nm})$, and $\mathrm{Au}(300 \mathrm{~nm})$ thin films were formed for insulation, strain gauge, shape control, and wiring, respectively.

\subsection{Principle of tactile sensing}

Figure 2(a) shows a conceptual illustration and a plot of resistance change of the strain gauge on a microcantilever when the normal force is applied to the hemispherical PDMS of the sensor. When an external force is applied on the sensor surface, microcantilevers are deflected with the deformation of the PDMS. As PDMS expands in the horizontal direction, microcantilevers are deflected upward and compressive stress is applied to the surface of the microcantilevers. The resistance of the strain gauge on the microcantilever decreases with increasing compressive stress. Thus, the resistance of the strain gauge depends on the applied normal force, as shown in Fig. 2(a). On the other hand, Fig. 2(b) shows impedance change of the Si layer measured through the insulation layer $(\mathrm{SiN})$ as a function of the applied force. The Si resistance seems to be changed by pressing force, however, the $\mathrm{Si}$ impedance change is hardly observed in Fig. 2(b). It is considered that the impedance change with applied force is too small to measure with the LCR meter (3532-50, Hioki) used in this work.

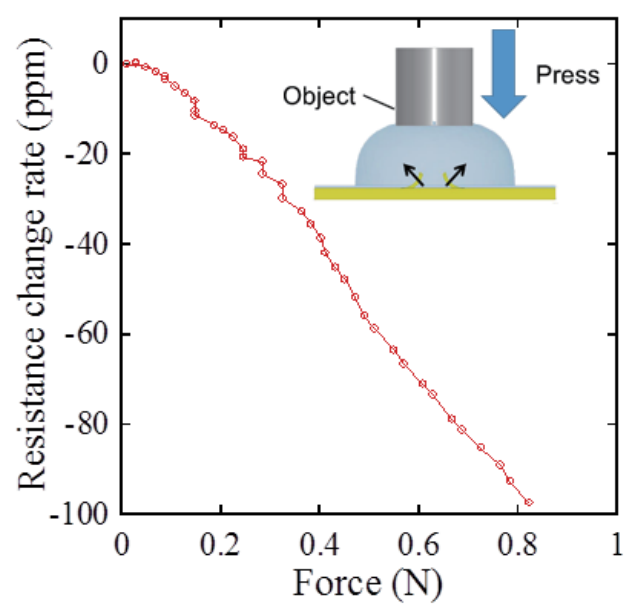

(a)

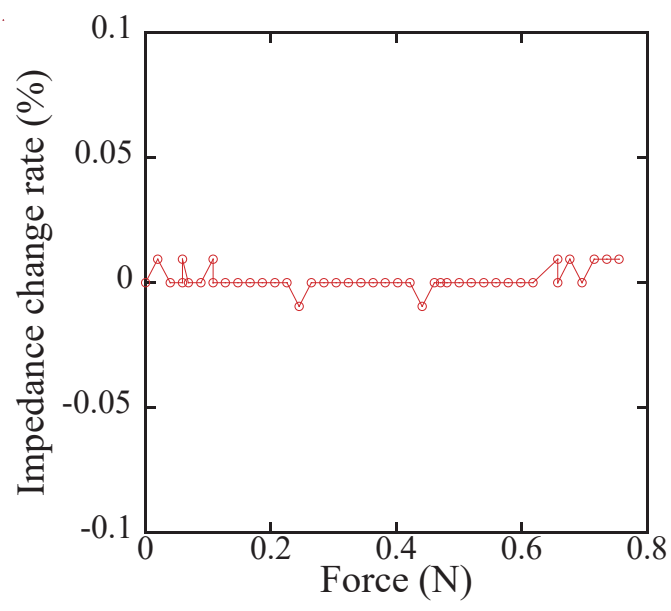

(b)

Fig. 2. (Color online) (a) The resistance change of the strain gauge on the microcantilever in the sensor and (b) the impedance change of the Si layer measured through the insulation layer as a function of the applied force. 


\subsection{Principle of visual sensing}

Figure 3(a) shows the relative AC impedance change of a Si layer at $5 \mathrm{MHz}$, which is the maximum measurement frequency of the LCR meter, because sensitivity of the impedance to light becomes larger at higher frequency ${ }^{(8)}$ as a function of illuminance of the white LED (LK-1WH-6, ELEKIT) light. The AC impedance between electrodes decreases with increasing current carrier density in the upper part of the Si layer of the SOI wafer based on the photoconductive effect. To confirm the thermal effect by the heat transfer or emission from the LED, thermal images of the measurement system when the LED was turned on $(10 \mathrm{~V})$ and off $(0 \mathrm{~V})$ were observed by an infrared camera (H2640, Nippon Avionics) as shown in Fig. 3(b). Although the temperature of the LED increases with applied voltage, the temperature change of the sensor is hardly observed. Thus, the intensity of light entering the sensor can be detected as an impedance change in the Si layer.

\subsection{Temperature dependences}

Figure 4 shows the relative resistance change of the strain gauge and impedance change in the Si layer as a function of temperature drop. When the sensor temperature decreases, the impedance through the Si layer increases and the $\mathrm{NiCr}$ strain gauge resistance also increases. This result means that the resistance change of the impedance in the $\mathrm{Si}$ layer and the resistance of $\mathrm{NiCr}$ strain gauge has a negative temperature coefficient. In this work, doping density of impurity in the Si wafer is low $\left(\sim 10^{15} \mathrm{~cm}^{-3}\right)$. It is well known that low-doped $\mathrm{Si}$ shows a negative temperature coefficient of resistance (TCR) because of an increase is thermally excited carriers. On the other hand, it is reported that some $\mathrm{NiCr}$ thin films show a near-zero or negative value of TCR in the case of around a 5:5 composition ratio, ${ }^{(13)}$ and we have a confirmed experimentally that the $\mathrm{Cr}-50 \% \mathrm{NiCr}$ thin film which is used in this work has a negative TCR.(14) It is considered that an amorphous crystal structure

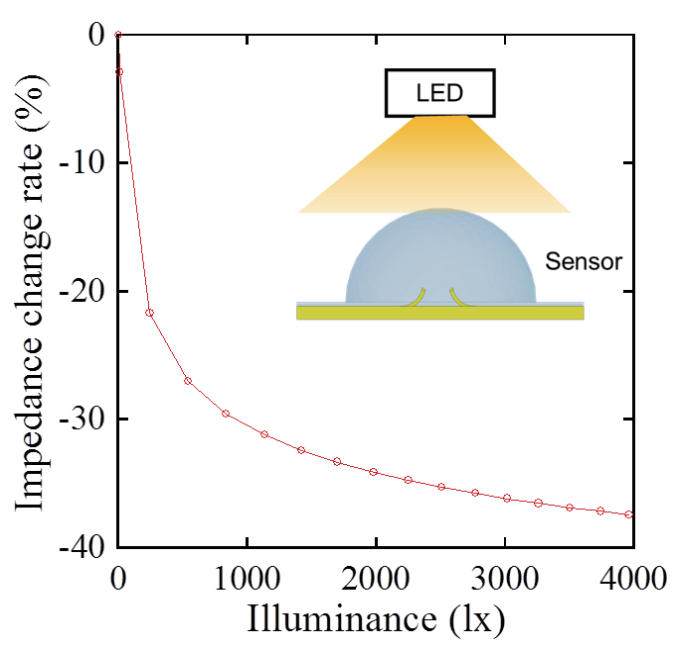

(a)

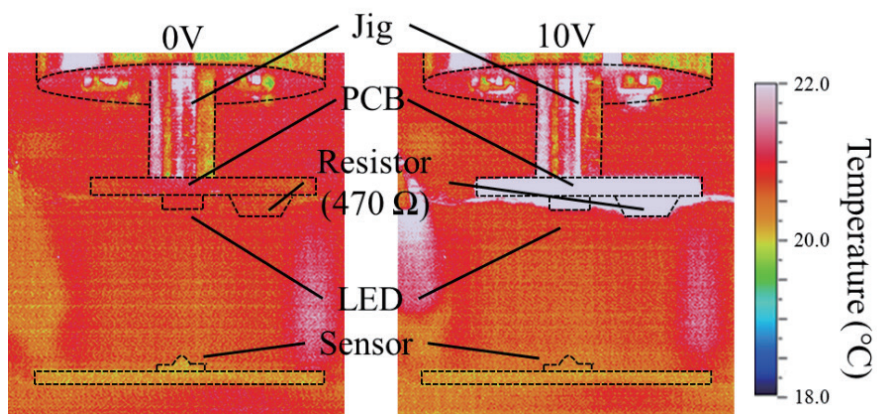

(b)

Fig. 3. (Color online) (a) Light illuminance dependence of the impedance change of the sensor and (b) thermal images when the LED was turned on $(10 \mathrm{~V})$ and off $(0 \mathrm{~V})$. 


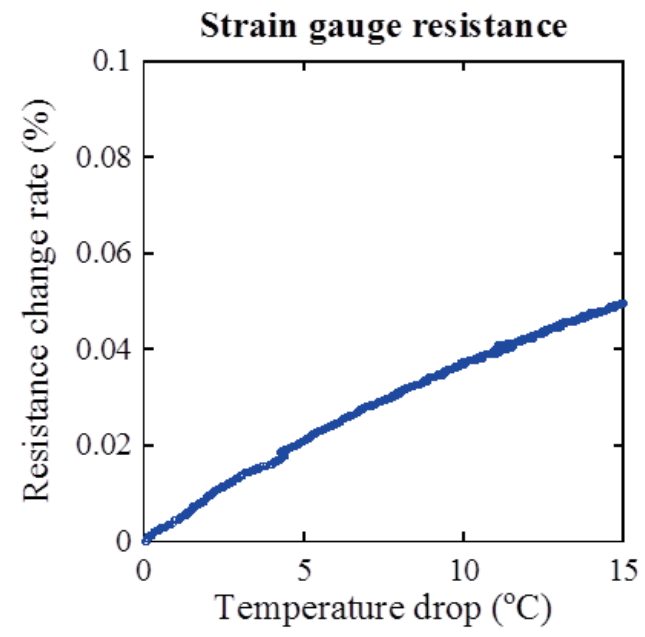

(a)

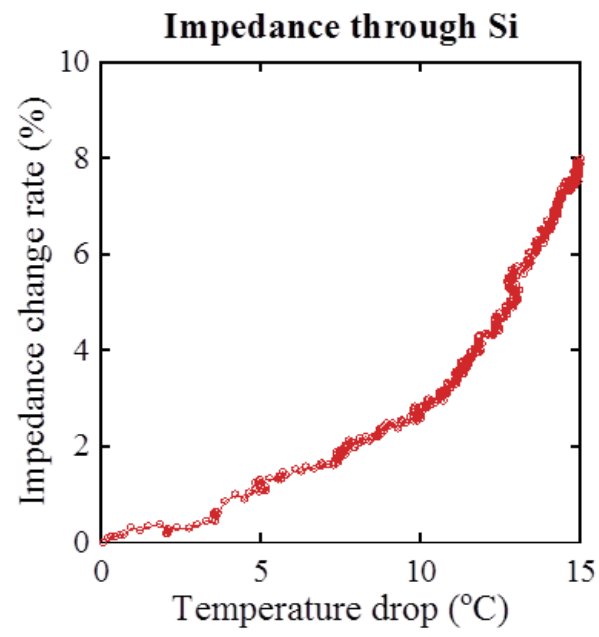

(b)

Fig. 4. (Color online) Temperature dependences of (a) the resistance change of the strain gauge and (b) the impedance change of the Si substrate.

causes this behavior. In our previous work, it has been shown by using an x-ray diffraction method that a deposited $\mathrm{NiCr}$ film become an amorphous crystal structure. ${ }^{(14)}$ In addition, the impedance change for temperature is considerably larger than the resistance change for temperature. The sensitivity of the impedance for the temperature of the Si substrate ranges from about 0.3 to $1.5 \% /{ }^{\circ} \mathrm{C}$. From the result in Fig. 4, it is found that both strain gauge resistance and impedance depend on temperature.

Here, the sensitivities to force, light, and temperature have some variations between fabricated sensors. Therefore, initial calibration by measurement of the sensitivity to force, light, and temperature as shown in Figs. 2-4 is necessary.

\subsection{Measurement system and target objects}

Table 1 shows the physical properties of the target objects used in this work. Three types of object, which have different densities and thermal characteristics, are characterized. Copper has a high thermal conductivity; on the other hand, wood and acrylic have low ones. Moreover, wood has a lower density than acrylic. Figure 5 shows a photograph of the measurement setup for texture characterization and target objects in this work. The fabricated MEMS sensor mounted on a commercially available printed circuit board was installed on an $x y$ horizontal motorized stage. The target object was attached to a $z$ vertical motorized stage through a brass jig and a 6-axis force sensor (Nitta Corp.) as a force reference. The target object was pressed on or approached the sensor surface by moving the $z$ stage in the vertical direction. The resistance and impedance changes were measured using a digital multimeter (R6581, Advantest) and an LCR meter (3532-50, Hioki), respectively. 
Table 1

Physical properties of target objects used in this work and the PDMS as the contacting part of the sensor. ${ }^{(15-17)}$

\begin{tabular}{lcccc}
\hline & Density $\left(\mathrm{g} / \mathrm{cm}^{3}\right)$ & $\begin{array}{c}\text { Specific heat } \\
\text { capacity }(\mathrm{J} / \mathrm{g} \cdot \mathrm{K})\end{array}$ & $\begin{array}{c}\text { Thermal } \\
\text { conductivity }(\mathrm{W} / \mathrm{m} \cdot \mathrm{K})\end{array}$ & $\begin{array}{c}\text { Young's modulus } \\
(\mathrm{GPa})\end{array}$ \\
\hline Copper & 8.96 & 0.38 & 398 & 129.8 \\
Wood & 0.4 & 1.3 & 0.16 & 7.85 \\
Acrylic & 1.18 & 1.4 & 0.21 & 3.14 \\
PDMS & 1.03 & 1.1 & 0.18 & 0.001 \\
\hline
\end{tabular}

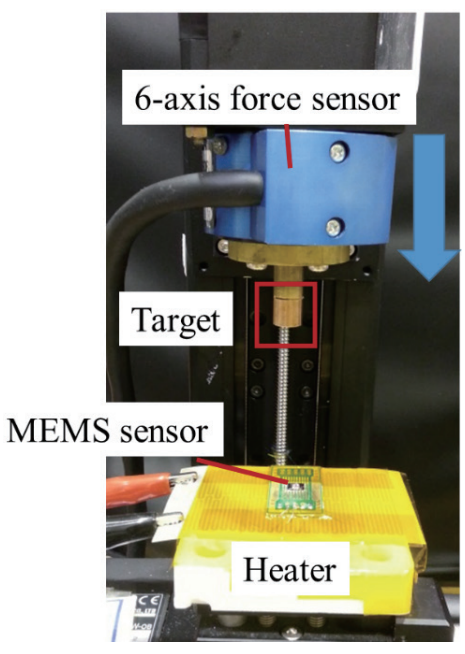

(a)

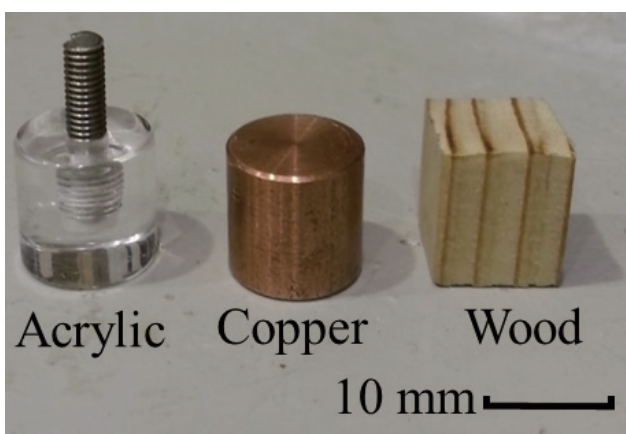

(b)

Fig. 5. (Color online) Photographs of (a) the measurement setup and (b) target objects.

Figure 6 shows a conceptual illustration of the press experiment. A sensor heated to about 40 ${ }^{\circ} \mathrm{C}$, which is similar to the deep body temperature of humans, was pressed vertically using the stage attached to each target object (R.T.) at $1 \mathrm{~mm} / \mathrm{s}$ until the contact force monitored by the 6 -axis force sensor achieved a constant value.

Figure 7 shows a conceptual illustration of the proximity experiment. The sample attached to the stage approached the sensor at $1 \mathrm{~mm} / \mathrm{s}$ from a $20 \mathrm{~mm}$ distance over the sensor surface in a darkroom. The LED (LK-1WH-6, ELEKIT) was located in a nearby position from the sensor and applied $10 \mathrm{~V}$. The reflected light from the target object was detected by the sensor as an impedance change of the Si layer.

\section{Results and Discussion}

\subsection{Press experiment}

Figure 8(a) shows resistance change as a function of elapsed time after contact. In the case of all target materials, the resistance of the strain gauge rapidly decreases immediately after contact, then the resistance of the strain gauge gradually increases. It is considered that the rapid decrease in resistance is caused by the contact force between the sensor surface and the objects, and the 


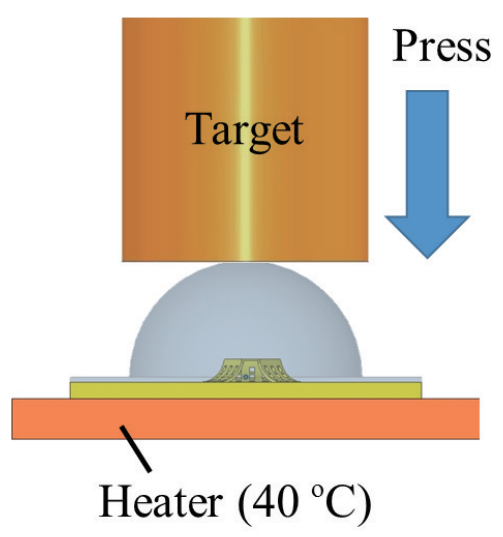

Fig. 6. (Color online) Conceptual illustration of press experiment.

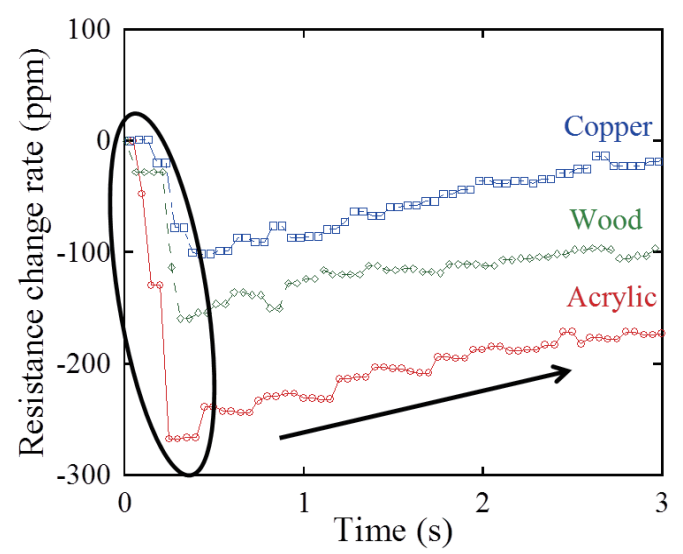

(a)

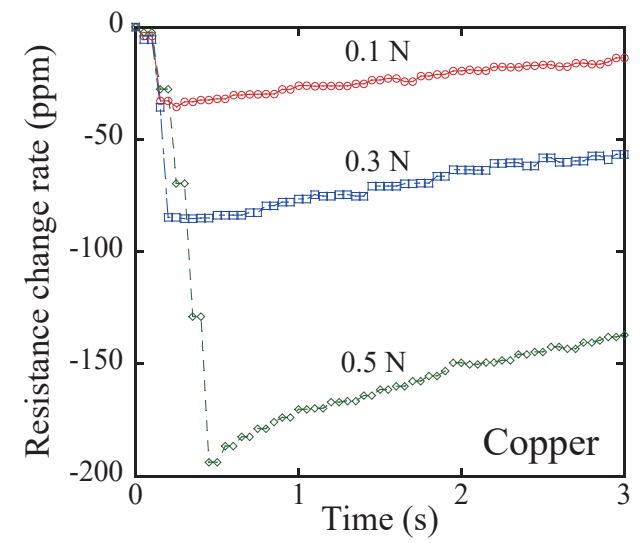

(c)

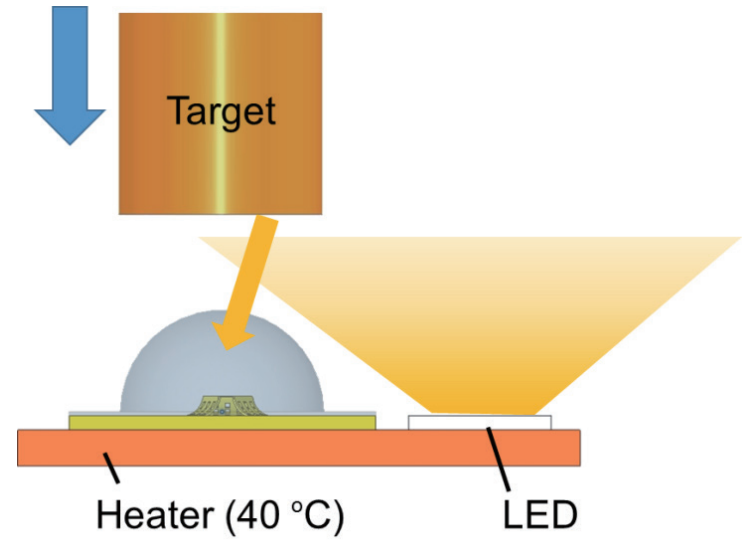

Fig. 7. (Color online) Conceptual illustration of proximity experiment.

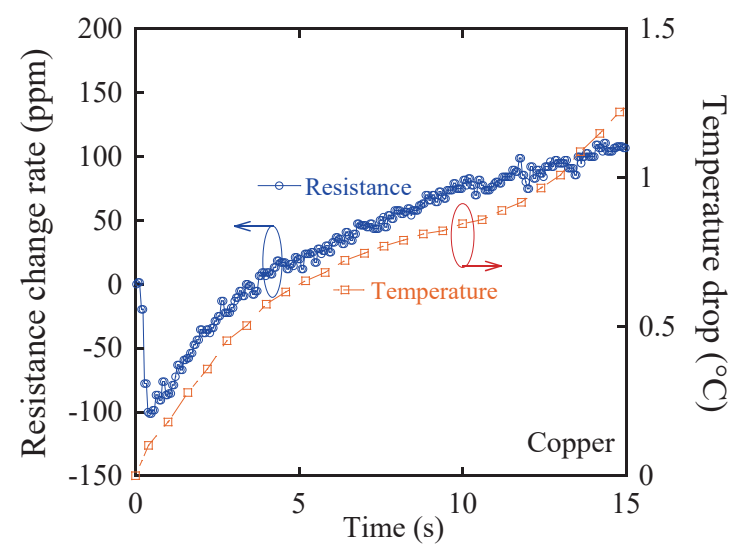

(b)

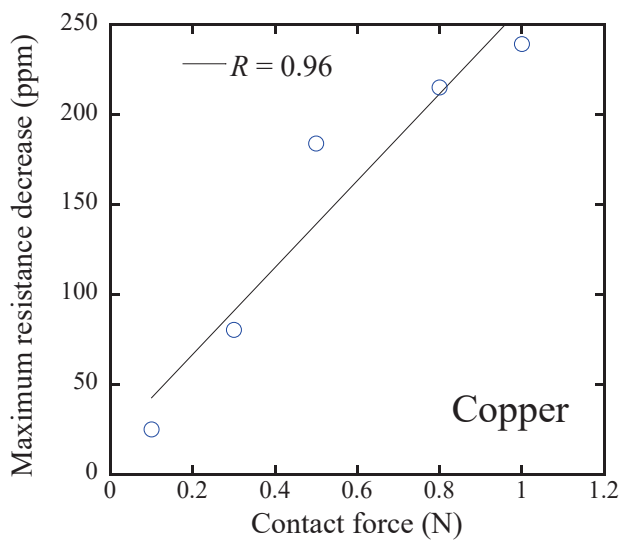

(d)

Fig. 8. (Color online) (a) Time dependence of the resistance change of the strain gauge after contact with various objects, (b) a comparison between time dependences of resistance change and the temperature drop of the sensor in the case of copper, (c) resistance change in the case of different contact forces, and (d) correlation between the maximum resistance decrease and contact force. 
gradual increase in resistance is due to the temperature drop caused by heat transfer from the sensor to the objects. Figure 8(b) shows the resistance change in the case of copper and the temperature drop of the sensor as a function of elapsed time. The gradual increase in resistance causes changes similar to the temperature drop; however, the rapid resistance decrease just after contact does not correspond with that of the temperature drop. Figures $8(\mathrm{c})$ and $8(\mathrm{~d})$ show the resistance change in the case of different contact forces and a correlation plot between the maximum resistance decrease and the contact force, respectively. The resistance decrease is obviously correlated with the contact force in the case of the same object. However, in Fig. 8(a), the initial rapid decrease in resistance is slightly different depending on material of the object although the contact force is the same. It is considered that this difference is due to the real contact area between the PDMS of the sensor and the object depending in a complicated fashion on the contact force, contact thermal conductance, and surface roughness. The correlation among these factors will be investigated in future work.

Figure 9 shows (a) the relative impedance change of the Si layer as a function of elapsed time after contact and (b) the impedance change and the temperature drop of the sensor as a function of

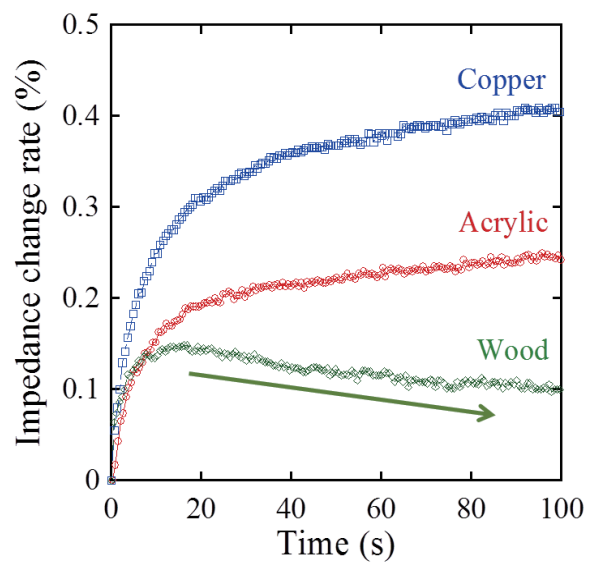

(a)

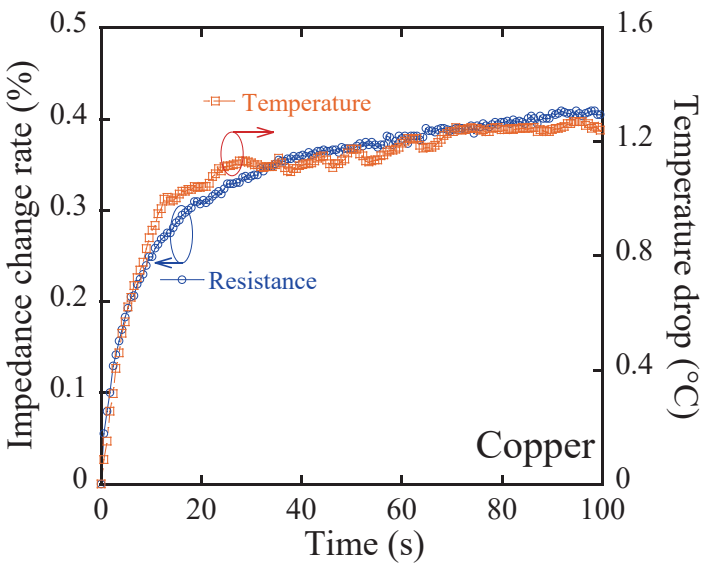

(b)

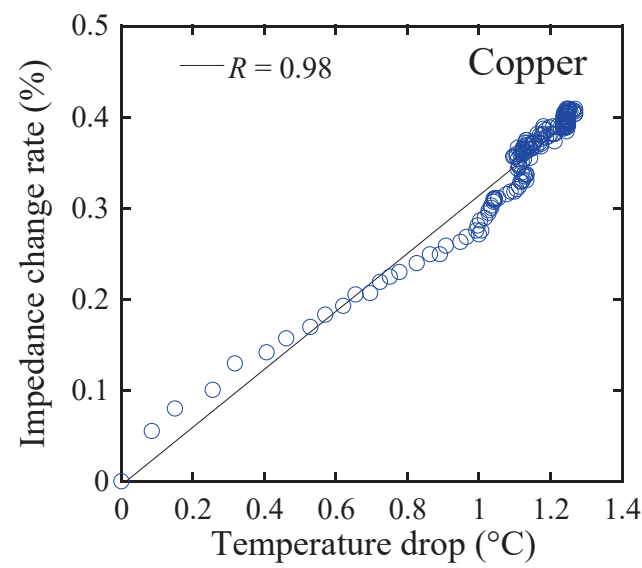

(c)

Fig. 9. (Color online) (a) Time dependence of the impedance change with temperature change after contact, (b) a comparison between time dependences of resistance change and the temperature drop of the sensor in the case of copper, and (c) a correlation plot between the impedance change and the temperature drop of the sensor. 
elapsed time. As shown in Fig. 9(a), in the case of copper, the impedance rapidly increases with a temperature change immediately after contact. In the case of acrylic, the impedance change is similar to that in the case of copper but the change is relatively small. On the other hand, in the case of wood, which has a lower density than the other materials, the impedance increases initially, but then decreases gradually. It is suggested that the impedance change is affected by the temperature drop caused by the differences in the density, the heat capacity (product of mass and specific heat), and the surface shape as well as the thermal conductivity of the object. The temperature drop at the interface between the sensor and the object, $\Delta T$, when the PDMS of the sensor contacts to the object is shown in the following equation, ${ }^{(18)}$

$$
\Delta T=\left(T_{s}-T_{o}\right) \frac{\sqrt{k_{o} \rho_{o} C_{o}}}{\sqrt{k_{s} \rho_{s} C_{s}}+\sqrt{k_{o} \rho_{o} C_{o}}},
$$

where $T_{s}\left(=40{ }^{\circ} \mathrm{C}\right)$ and $T_{o}\left(=23{ }^{\circ} \mathrm{C}\right)$ are the initial temperature of the sensor and the object, respectively, and $\sqrt{k_{s} \rho_{s} C_{s}}$ and $\sqrt{k_{o} \rho_{o} C_{o}}$ are the thermal effusivity of them calculated from their thermal conductivity $\left(k_{s}, k_{o}\right)$, density $\left(\rho_{s}, \rho_{o}\right)$, and the specific heat capacity $\left(C_{s}, C_{o}\right)$, respectively. From the values shown in Table 1, the calculated $\Delta T$ for copper, wood, and acrylic give 16.8, 6.6, and $9.6^{\circ} \mathrm{C}$, respectively. Ratios of calculated temperature drop for wood and acrylic to that for copper are approximately 0.4 and 0.6 , respectively. If the temperature drop of the sensor is proportional to $\Delta T$, the ratio of impedance change to temperature drop between these targets is similar to the ratio of $\Delta T$. The ratios of the maximum impedance change for wood and acrylic to that for copper are approximately 0.4 and 0.6 , respectively. Therefore, the impedance change corresponds quantitatively to $\Delta T$. Furthermore, it is confirmed that the impedance change reflects the temperature drop of the sensor, and there is a high correlation $(R=0.98)$ between the impedance change and the temperature drop as shown in Figs. $9(\mathrm{~b})$ and $9(\mathrm{c})$, respectively.

\subsection{Proximity experiment}

Figure 10 shows relative impedance change as a function of the distance between the sensor surface and the target object. As the object approaches the sensor, the impedance gradually decreases. The impedance change reaches its maximum decrease at a distance of around $5 \mathrm{~mm}$, and then the impedance increases because the incident light intensity on the sensor decreases as the object approaches more closely to the sensor surface. On the other hand, without a probe light, impedance changes are hardly observed in the cases of both copper and wood, as shown in Fig. 10(a). However, as shown in the enlarged graph [Fig. 10(b)], it is found that the impedance slightly increases near the sensor without light. It is considered that the sensor temperature slightly decreased because of contactless heat transfer through thermal convection and thermal radiation or photogenerated carriers excited by thermal-radiated infrared rays. This result indicates a possibility of non-contact detection of the approximate temperature of an object. Figure 11 shows the maximum impedance decrease in Fig. 10(a). It is possible to clearly distinguish copper and wood on the basis of these impedance changes caused by the differences in surface light reflectance, as shown in Fig. 11. 


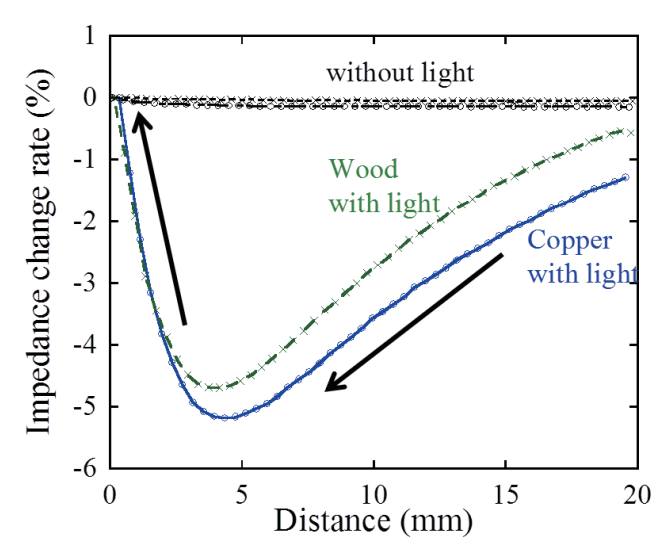

(a)

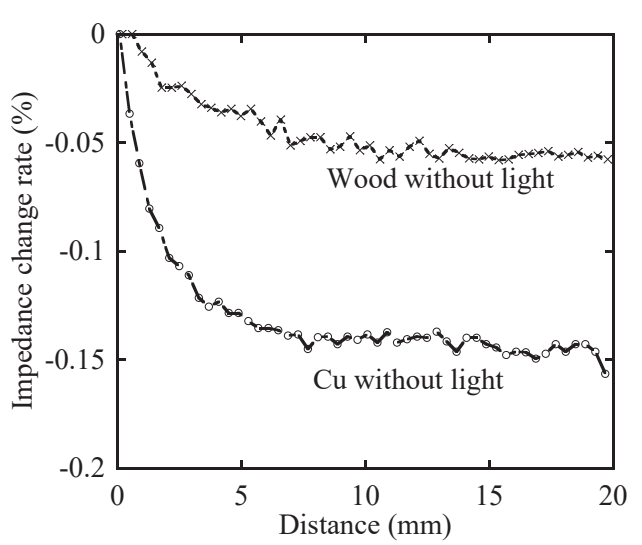

(b)

Fig. 10. (Color online) (a) Impedance change of Si layer as a function of proximity distance between the sensor surface and the object and (b) enlarged graph in the case of without light.

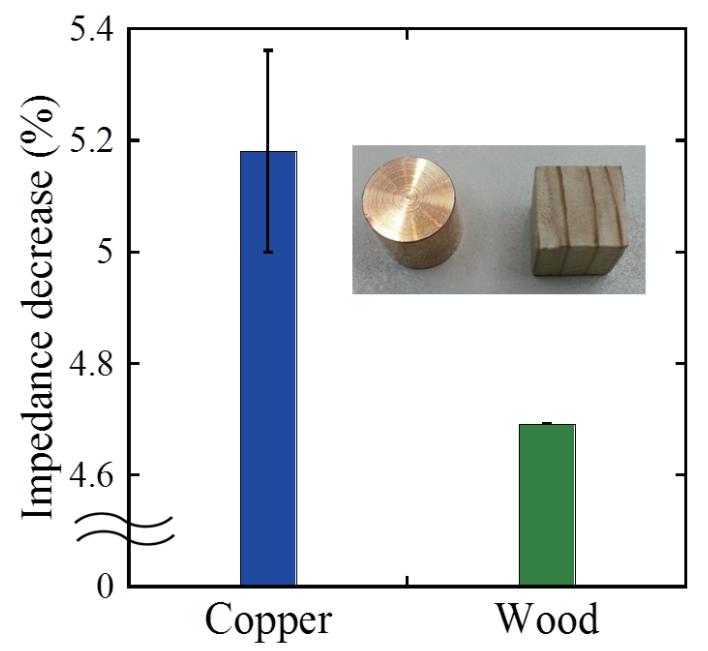

Fig. 11. (Color online) Maximum impedance decrease in the proximity experiment of copper and wood.

\section{Conclusions}

A MEMS sensor with a strain gauge on microcantilevers and impedance of the Si layer has been experimentally applied to the quantitative measurement of the multimodal (including tactile, light, and warm/cool sensations) texture of an object. In the experiment of pressing an object to the sensor surface, it is possible to detect the contact of the object on the case of the resistance change of the strain gauge and the impedance change of the Si layer due to a temperature drop, which depends on the heat characteristics and the density of the object. In the proximity experiment of moving the object to the sensor surface, the impedance change caused by reflected light from the object depends on the light reflectance of the object. In summary, the changes in the resistance 
of a strain gauge and the impedance of a Si layer depend on the physical interactions between the sensor and the object caused by their relative movements. It is promising to measure complex surface texture including tactile, light, and warm/cool sensations separately using the correlation of sensor output with active measurement similar to human exploratory behavior during object discrimination. For example, light reflectance of the object is detected by the impedance change before contact, then the warm/cool sensation is detected by the impedance or resistance change immediately after contact, and then the contact force is detected by the resistance change which is obtained by pressing when the resistance change caused by the temperature change is saturated.

In future work, dependence of the sensor outputs on environmental conditions such as ambient temperature and humidity which affect the texture sensation will be investigated. Furthermore, the sensor outputs will be compared with a result of the human sensory evaluation or the electroencephalogram analysis.

\section{Acknowledgement}

A part of this work was supported by a research grant from The Union Tool Scholarship Foundation.

\section{References}

1 Y. Shimizu, T. Sadoyama, M. Kamijo, S. Hosoya, M. Hashimoto, and T. Otani: Int. J. Cloth. Sci. Tech. 16 (2004) 32.

2 K. Kim, M. Takatera, A. Zhu, and T. Tsuyoshi: Autex Res. J. 15 (2015) 67.

3 A. M. Mazid and R. A. Russell: 2006 IEEE Conf. Robotics, Automation and Mechatronics (2006) 1.

4 K. Kuramitsu, T. Nomura, S. Nomura, T. Maeno, and Y. Nonomura: Chem. Lett. 42 (2013) 284.

5 S. Okamoto, H. Nagano, K. Kidowa, and Y. Yamada: Int. J. Affective Eng. 15 (2016) 11.

6 M. Sohgawa, D. Hirashima, Y. Moriguchi, T. Uematsu, W. Mito, T. Kanashima, M. Okuyama, and H. Noma: Sens. Actuators, A 186 (2012) 2.

7 M. Sohgawa, K. Watanabe, T. Kanashima, M. Okuyama, T. Abe, and H. Noma: 2014 IEEE Sensors Proc. (IEEE, Valencia, 2014) p. 1706.

8 M. Sohgawa, A. Nozawa, H. Yokoyama, T. Kanashima, M. Okuyama, T. Abe, H. Noma, and T. Azuma: 2014 IEEE Sensors Proc. (IEEE, Valencia, 2014) p. 1749.

9 K. Takahashi, T. Abe, M. Okuyama, H. Noma, and M. Sohgawa: 2015 IEEE Sensors Proc. (IEEE, Busan, 2015) p. 699.

10 X. Chen, F. Shao, C. Barnes, T. Childs, and B. Henson: Int. J. Design 3 (2009) 67.

11 H. Shirado and T. Maeno: World Haptics Conference (IEEE, Pisa, 2005) p. 629.

12 H. Yokoyama, M. Sohgawa, T. Kanashima, T. Azuma, M. Okuyama, and H. Noma: Jpn. J. Appl. Phys. 52 (2003) 06GL08.

13 S. Yamashita, H. matsubara, and T. Fukami: J. Surf. Finish. Soc. Jpn. 50 (1999) 41 (in Japanese).

14 H. Tachibana, S. Kamanaru, H. Matsuura, M. Sohgawa, T. Kanashima, M. Okuyama, K. Yamashita, M. Noda, and H. Noma: The Papers of Tech. Meet. Phys. Sens., IEEJ (2009) pp. 97-102 (in Japanese).

15 T. Ogi: Chronological Scientific Tables, ed. National Astronomical Observatory (Maruzen Co., Tokyo, 2010) Chap. 4.

16 Japan Society of Mechanical Engineers: JSME Textbook Series Heat Transfer (Maruzen Co., Tokyo, 2011) Chap. 1 (in Japanese).

17 D. Erickson, D. Sinton, and D. Li: Lab Chip 3 (2003) 141.

18 Japan Society of Mechanical Engineers: JSME Textbook Series Heat Transfer (Maruzen Co., Tokyo, 2011) Chap. 2 (in Japanese). 